\title{
La elección de la teoría: Prácticas científicas en filosofía y epistemología de la imaginación.
}

\author{
Luis Mauricio Rodríguez Salazar \\ Docente Investigador en el Centro de Investigaciones Económicas Administrativas y \\ Sociales del Instituto Politécnico Nacional. (CIECAS-IPN) \\ Miembro del Sistema Nacional de Investigadores Nivel 1 (SNI-CONACyT- \\ México) \\ Guillermo Samuel Tovar Sánchez \\ Licenciado en Psicología por la Benemérita Universidad Autónoma de Puebla. \\ Maestro en Ciencias en Metodología del Ciencia en el Centro de Investigaciones \\ Económicas Administrativas y Sociales (CIECAS-IPN) \\ Estudiante del Doctorado en Investigación en Ciencias Sociales por la Facultad \\ Latinoamericana de Ciencias Sociales (FLACSO-México) \\ Becario Conacyt \\ guillermo.sts@gmail.com
}

\section{Resumen}

La elección de la teoría, como lo expuso Kuhn, es un tema que ha sido objeto de discusión en la filosofía de la ciencia y en la epistemología, traída ahora a la metodología de la ciencia. Kuhn argumenta que en las ciencias sociales esta polémica suscitó muchos problemas sobre si una ciencia social es o no científica. Ahora el problema es saber si un tema es o no propio de la metodología de la ciencia. En ese tenor, el objetivo de este trabajo es analizar dos propuestas epistemológicas contemporáneas que se titulan epistemología naturalizada social y la epistemología de la imaginación, ambas como fundamento del método en la práctica científica. Por lo tanto, en los dos primeros apartados se describen ambas propuestas epistemológicas en términos de sus componentes más relevantes, así como sus fundamentos característicos bajo los criterios de Kuhn sobre elección de la teoría. La propuesta es que, para cualquier proceso metodológico, se tiene que hacer explícita la teoría que sustenta el método, tema de discusión en la Maestría en Ciencias en Metodología de la Ciencia que se imparte en el Instituto Politécnico Nacional.

Palabras clave: epistemología, filosofía de la ciencia, práctica científica, epistemología de la imaginación 


\title{
THE CHOICE OF THEORY: SCIENTIFIC PRACTICES IN PHILOSOPHY AND EPISTEMOLOGY OF IMAGINATION
}

\begin{abstract}
The choice of theory, as Kuhn put it, is a subject that has been the subject of discussion in the philosophy of science and in epistemology, now brought to the methodology of science. Kuhn argues that in the social sciences this controversy raised many problems about whether a social science is scientific or not. Now the problem is to know whether or not a subject belongs to the methodology of science. In this vein, the objective of this work is to analyze two contemporary epistemological proposals that are entitled social naturalized epistemology and the epistemology of imagination, both as the foundation of the method in scientific practice. Therefore, the first two sections describe both epistemological proposals in terms of their most relevant components, as well as their characteristic foundations under Kuhn's criteria on the choice of theory. The proposal is that, for any methodological process, the theory that supports the method must be made explicit, a subject of discussion in the Master of Science in Science Methodology taught at the National Polytechnic Institute.
\end{abstract}

Keywords: epistemology, philosophy of science, scientific practice, epistemology of imagination

Artículo recibido: 4. octubre. 2020 Aceptado para publicación: 24. Octubre. 2020 Correspondencia: guillermo.sts@gmail.com Conflicto de interés: Ninguna que declarar 


\section{Introducción}

La elección de una teoría es un tema frecuentemente polémico en las discusiones filosóficas y epistemológicas. Por un lado se defiende una postura totalmente racional o racionalista de ella, apelando a la objetividad en términos clásicos (universal y necesaria) sin que ello signifique una comprehensión global de su génesis. En una segunda perspectiva, se sostiene que tal elección no es una cuestión meramente objetiva, sino que involucran aspectos subjetivos o propios del sujeto que elige tal o cual teoría. Esta última opción es la que desarrolla Kuhn, especialmente en el penúltimo capítulo de su libro La tensión esencial: estudios selectos sobre la tradición y el cambio en el ámbito de la ciencia (1977/1996).

En dicho texto, Kuhn realiza toda una defensa de su postura argumentando que sus críticos han malentendido su propuesta hasta en un doble sentido. Por un lado, la crítica se puede enmarcar en la discusión sobre los contextos de justificación y descubrimiento, misma que sus críticos sostienen que donde comienza la segunda termina el trabajo de la filosofía de la ciencia y comienza una discusión más sociológica o psicológica. En ese sentido, Kuhn revira diciendo que quien usualmente utiliza dicho planteamiento confunde la discusión con la que refiere la pedagogía de la ciencia. Dicho de otra manera, que los argumentos que diferencian ambos tipos de contexto se sustentan en lo que normalmente se exponen en los libros de texto, que instruyen a los estudiantes de ciencia a través de ejemplos de cómo se fueron alcanzando los objetivos o descubrimientos científicos.

En consecuencia, dicha discusión desembocó en los últimos años en el problema de si la ciencia es valorativamente neutra, trascendiendo los contextos de descubrimiento o de justificación. En ese sentido, Olivé (2006) expone dos tesis que clarifican la discusión como sigue:

1. La ciencia y la tecnología no plantean ningún problema o cuestión ética, sólo sus aplicaciones. Es decir, que son valorativamente neutras. Esta concepción se basa en la distinción entre hechos y valores. Siendo los hechos los eventos concretos de la realidad y los valores los juicios que determina el interés sobre un objeto. Así la ciencia y la tecnología se encargan únicamente de describir y explicar hechos no de emitir juicios de valor sobre ellos. 
2. La ciencia y la tecnología sí plantean problemas éticos, tanto en su aplicación como en su concepción. Es decir, son valorativamente NO neutras. En este sentido, se concibe a la ciencia y la tecnología como sistemas de acciones intencionales: "como sistemas que incluyen agentes que deliberadamente buscan ciertos fines, en función de determinados intereses, para la cual ponen en juego creencias, conocimientos, valores y normas" (pp. 85-86)

En ese sentido, la ciencia se desarrolla por las personas y estas buscan cumplir sus fines. Esto implica que para el desarrollo y la producción de conocimiento es importante la autonomía de la ciencia, lo que, a su vez, implica que las maneras en las que se practica una disciplina son de vital importancia para la organización social de la ciencia. Es decir,

La autonomía epistémica significa que el conocimiento científico y sus aplicaciones se generan dentro de comunidades científicas y tecnológicas por medio de prácticas, es decir, de complejos de acciones que tienen sus propios y específicos valores y normas para organizar el trabajo, así como para aceptar o rechazar sus productos (hipótesis, modelos, técnicas, tecnologías) (Olivé, 2008: 41)

Aunado a lo anterior, Risjord (2014), en su libro "Filosofía de las ciencias sociales" plantea el problema sobre ¿cuáles son los valores que juegan un papel en el desarrollo de la ciencia?, y ¿cómo estos valores tienen influencia en la práctica científica? Para resolver estas preguntas, plantea que existen dos tipos de valores en la ciencia, los constitutivos por una parte y los contextuales por otra (que se asemejan al contexto de justificación y descubrimiento).

En cuanto a los primeros, se refiere a los aspectos que son parte del progreso científico desde una perspectiva moderada de los valores. Estos valores son epistémicos toda vez que ayudan a construir el conocimiento y están ajenos a la influencia de las dimensiones política, social, económica, etcétera.

Por lo tanto, que los valores contextuales se refieren específicamente a los valores que se relacionan con los intereses de los agentes, a los hechos o eventos históricos concretos que contextualizan el progreso científico. Sin embargo, el mismo Risjord menciona que el debate contemporáneo está en discernir si los valores morales y políticos son 
constitutivos de la ciencia o no. Para ello ofrece una cantidad de ejemplos donde las presiones sociales, los intereses políticos y otros, influyen en la constitución del progreso científico.

En ese sentido, en este trabajo se sostiene que ambos componentes son sustanciales para dar cuenta de la estructura de la ciencia y que intervienen en la elección de la teoría. Esto nos lleva a considerar la segunda respuesta de Kuhn a sus críticos: el tema de la objetividad y subjetividad para elegir la teoría que le de sustento a una propuesta metodológica cualquiera, que resulta de gran relevancia en una Maestría en Ciencias en Metodología de la Ciencia.

Para Kuhn (1977/1996) la subjetividad que él plantea en su obra sobre la elección de teoría fue malinterpretada por los filósofos, considerándola como una simple elección individual de gustos por tal o cual teoría. Sin embargo, el historiador de la ciencia anglosajón argumenta que no se trata de eso, sino que, recurriendo a Kant, es un tema de juicios estéticos. Es decir, cuando un científico evalúa una teoría es a través de una serie de juicios sobre su constitución lo que permite, en cierto sentido, tal o cual dirección en su elección. Estos juicios deben llevarse a crítica por su comunidad.

El argumento de Kuhn (1977/1996: 362) es que "la elección de la teoría indica algunas de las limitaciones de la objetividad, pero sin aislar los elementos llamados con propiedad subjetivos". De esto, aquí se interpreta que la elección de la teoría científica, en tanto que juicio estético, conduce a una comunicación entre lo objetivo de la teoría y lo subjetivo del sujeto. Así, dice Kuhn, para la persona de ciencia que guste de explicar los movimientos celestes, podrá poner a prueba dos teorías que expliquen tal o cual fenómenos con base en sus sustentos racionales. Sin embargo, si ambas teorías explican con, más o menos, la misma eficiencia su elección puede depender de su formación o de criterios propios de su personalidad. Empero, alguien que se decante por una posición terraplanista para explicar los movimientos celestes difícilmente encontrará eco en la comunidad científica.

En ese tenor, Kuhn propone cinco características de la ciencia normal que no difieren a los tradicionales en cuanto a forma o función. Estos son precisión, coherencia, amplitud, simplicidad y fecundidad. Desde un enfoque tradicionalista, estas características son 
usualmente reglas estáticas desde una perspectiva tradicional, en cambio desde la postura de Kuhn estas son consideradas como valores que pueden variar en cuanto su peso de elección. Así, para algunas disciplinas es más importante la precisión que la simplicidad, o es deseable contar con coherencia lógica que con la fecundidad de la teoría.

En resumen, lo que Kuhn hace es una crítica hacia los modelos algorítmicos de la elección de la teoría que, si bien son útiles e interesantes, no dan respuesta cabal al problema de la elección. De ese modo, una de las formas de elegir tal o cual teoría radica en su comprehensión objetiva y en las características subjetivas en tanto que juicios estéticos. Es decir, toda elección debe ser sujeta a crítica después de su comprehensión y puesta en relación al campo material que se propone explicar.

De ese modo, lo que en este trabajo se defiende surge a partir del trabajo de tesis de maestría del autor, en la que se analiza la acción del sujeto como prácticas que coadyuvan a la organización social, intentando fundamentar epistemológicamente la psicosociología desde un marco de la acción. Pero no sólo eso, sino también cómo la psicosociología puede ser de utilidad para criticar los componentes epistemológicos. Como lo plantea Piaget (1967/1979b:36) "la psicosociología puede, pues, proporcionarle a la epistemología datos utilizables para la solución de los problemas generales”.

En un segundo momento y como estudiante de metodología de la ciencia de formación en psicología con orientación hacia lo social, resulta especialmente importante realizar esta argumentación epistemológica. Pues es lamentable que en la formación se sigan utilizando juicios que competen más hacia aspectos metódicos y no los propios de la razón. Es decir, pareciera que existe una logotomía (Rodríguez-Salazar \& Rosas-Colín, 2016: 55) en la metodología de las actuales disciplinas, pues por esta última la entienden más cercano a los modelos algorítmicos que critica Kuhn, además, se materializan en forma de protocolos rígidos sin considerar la capacidad creadora de la razón.

Seguido de lo anterior, Kuhn (1962/1985: 247-248) ejemplifica lo que se intenta demostrar al mencionar que "hay hombres que pretenden que la psicología es una ciencia, debido a que posee tales y cuales características. Otros, al contrario, arguyen que esas características son innecesarias o que no son suficientes para convertir a ese campo en ciencia." a lo que continúa diciendo que el problema es que se están haciendo las 
preguntas equivocadas y más bien es necesario preguntarse “¿por qué no progresa mi campo del mismo modo que lo hace, por ejemplo, la física? ¿Qué cambios de técnicas, de métodos o de ideología lo harían capaz de progresar de esa forma?"

Lo que pone de manifiesto Kuhn es la falta de acuerdo en las ciencias sociales. Es decir, que no existe una teoría general que englobe a todas las demás como se hace en física. Que es una especie de etapa temprana de las ciencias sociales. Mientras que las ciencias naturales tienen clara la forma de elegir su problema, independiente de su importancia social, las ciencias sociales no pueden omitir tal exigencia. Por consiguiente, este trabajo representa una contribución a la discusión en ese sentido, no para legitimar las ciencias sociales como ciencia, sino, más bien, para encontrar pistas hacia dónde dirigir la discusión en tanto que su estructura epistémica.

Por tanto, como ya se señaló, este trabajo consiste en llevar a cabo una descripción de dos propuestas epistemológicas contemporáneas, bajo la orientación kuhniana de elección de teoría, para observar sus puntos de acuerdo, así como sus diferencias. Esto invitará a establecer cuáles son los posibles caminos a tomar para profundizar en fundamentos epistémicos contemporáneos para las ciencias sociales en general y para la psicosociología en particular desde la metodología de la ciencia.

\section{La práctica científica desde la filosofía}

Con base en lo anterior, se vuelve necesario entablar una discusión entre dos enfoques contemporáneos que abordan el tema epistemológico de la acción en la ciencia desde diferentes enfoques. En primer lugar, se comienza con el siguiente cuestionamiento de Martínez (et. al., 2011; 2005): ¿Es posible que los aspectos "externos” de la ciencia, tengan algún valor epistémico para la ciencia?, es decir, ¿las prácticas científicas pueden servir como un recurso para dar cuenta de la estructura normativa de la ciencia?

Para Martínez (2005; 2009; Martínez et. al., 2011) las prácticas tienen un valor epistémico toda vez que influyen en la conformación de normas que brindan confiabilidad y validez a la ciencia. Para dar cuenta de esto, el autor que se cita, sostiene que la filosofía de la ciencia tradicionalmente se ha centrado en teorías, es decir, que el objeto de la filosofía es independiente de cualquier contexto de descubrimiento. Por tanto, en la introducción que realizan Huang y Martínez para la compilación de 2011 cuestionan a la filosofía 
tradicional centrada en teorías y proponen un proyecto filosófico centrado en prácticas en dos tesis:
a. [...] una filosofía centrada en teorías es una filosofía muy limitada, que pasa por alto muchos factores que entran en la construcción del conocimiento científico, y que estos factores son explicables solo si tomamos las prácticas como un recurso que nos permite explicar la diversidad y dinámica del conocimiento científico para arrojar luz sobre controversias epistémicas importantes. (p. 9)

b. La segunda tesis que promovemos insiste en que el rechazo de una filosofía de la ciencia centrada en teorías no implica, como algunos constructivistas asumen, el rechazo de la centralidad de la epistemología para entender lo que es la ciencia. (p. 9)

La premisa [a] nos sugiere un proyecto filosófico que advierte la necesidad de incluir el concepto de práctica como recurso explicativo de la normatividad de la ciencia. Mientras que la premisa $[b]$ rechaza cualquier relativismo radical que pueda surgir a partir de considerar las prácticas como recurso explicativo. Esto implica considerar un concepto tan polisémico como el de práctica dentro de un marco epistemológico que reduzca toda dinámica a lo social o a una mera estructura subjetiva.

En ese sentido, la propuesta que se denomina epistemología naturalizada social, hace énfasis en que lo cognitivo tiene un origen irreductiblemente social y, por tanto, el concepto de práctica es fundamental para explicar la ciencia. En efecto, dicho concepto puede servir como un recurso explicativo del cómo se construye el conocimiento humano, puesto que este último no sólo se compone de representaciones, sino, que “...incorpora actos y capacidades de comprensión o entendimiento...” que son “... articulados en las prácticas sociales tanto dentro como fuera de los sujetos, en la interacción de los agentes con su entorno." (Martínez \& Huang, 2011:41)

Así este tipo de epistemología implica considerar el contexto en el que el agente construye el conocimiento con base en reglas heurísticas antes que abstractas. Para dichos autores, estas reglas heurísticas son tomadas como unidades de análisis y las contextualiza para 
entender la racionalidad humana. En otras palabras, la racionalidad se explica a través de acomodos heurísticos que se encuentran contextualizados en un momento determinado.

Por otro lado, Martínez (p. 43) critica la racionalidad de medios a fines tradicional y propone dos tesis críticas:

\section{1. "[...] la racionalidad científica va más allá de la elección de teorías."}

2. "[...] el establecimiento y la aplicación de las normas de medio/fin requieren una serie de factores contextuales explicables únicamente por ciertos conceptos de práctica.”

De ese modo, la racionalidad es más que lo que propone el argumento de la filosofía tradicional sobre formalizar el conocimiento, puesto que la racionalidad puede abarcar el terreno inexplorado de las prácticas para dar cuenta de dicha estructura normativa epistémica. Este nuevo proyecto requiere tomar en serio el contexto y los procesos de interacción del agente que construye conocimiento.

De esa manera, la práctica científica tiene un valor epistémico toda vez que el conocimiento científico

[...] no viene de reglas universalmente aplicables, sino, tiene que ver con la manera en la que se codifican, individualizan y clasifican tipos de actividades, en particular interacciones con otros agentes y con lo que se reconoce como recursos materiales, que toman en cuenta de manera implícita las limitaciones de tiempo y capacidad de procesamiento de la información que tenemos en una situación dada. (pp. 45-46)

En el capítulo en solitario de Martínez (2011: 219) de la obra referenciada, acota que las representaciones que son epistémicamente importantes se dividen en dos propuestas que entienden a la cognición articulada en prácticas de manera distinta. Por un lado, tenemos la visión cartesiana y por otro la interaccionista. La primera entiende a la práctica como un agregado de procesos individuales. El segundo, entiende que las prácticas científicas “... estructuran una variedad de normas cognitivas y estrategias de investigaciones que no puede entenderse como algo que es un mero epifenómeno de interacciones entre 
estructuras cognitivas propias de los individuos." Es decir, que "Para un interaccionista, la cognición incluye tanto procesos individuales como sociales.” (p. 221)

Por tanto, una de las tesis centrales del interaccionismo para Martínez es que

[...] las diferentes interacciones entre procesos cognitivos constituyen muchas veces contextos relativamente estables en donde tiene lugar la identificación e individuación de diferentes tipos de procesos que entran en juego en la cognición. Es más, un interaccionista arguye que los procesos sociales cognitivos irreductibles están coordinados, y en un grado importante constituidos, por prácticas científicas. (p. 221)

Por tanto, este enfoque epistemológico sostiene que, para explicar el conocimiento, hay que considerar la manera en que se estructuran los esquemas mentales a partir de las "representaciones" "situadas" (contextualizadas) y que estas formas de "estructuración" las podemos encontrar en la práctica misma del agente.

Para entender esto, entonces, se sigue necesariamente la explicación de la tesis del paralelismo ramificado, misma que explica Martínez (2011) como sigue:

Según la tesis del paralelismo ramificado la cognición no requiere una coordinación centralizada respecto a la cual pueda en última instancia hablarse de un tipo de proceso "interno" en oposición a uno "externo" (uno que no estaría sujeto a esa coordinación centralizadora). Más bien, esta tesis apoya la idea crucial a modelos interaccionistas de que la cognición tiene lugar a través de procesos "paralelos" que se ramifican en capas formadas por diferentes actividades de los que emerge la agencia. Así, el sentido en el que podemos entender que la cognición es distribuida es el sentido en el que podemos decir que la agencia es distribuida y que las "actividades" constituyen un nexo entre agencia y cognición (p. 223)

Con esto, se intenta poner de manifiesto que el interaccionismo que se propone, como proyecto filosófico, trata de entender a la agencia y la cognición distribuida en relaciones simultáneas y traslapadas en su devenir. Así la cognición y la agencia toman una misma 
posición a la hora de explicar los procesos epistémicos que explican el desarrollo del conocimiento.

Para resumir hasta ahora el proyecto filosófico centrado en prácticas, podemos argumentar que su objetivo es observar la práctica científica como un recurso explicativo de la estructura normativa-epistémica de la ciencia; que el concepto de práctica no se puede reducir a otros que expliquen con mejor o misma eficacia (de aquí su necesidad); que se fundamenta en la tesis del paralelismo ramificado y que, por tanto, la agencia y la cognición forman parte de un mismo proceso en la adquisición de conocimiento.

Como lo sostienen en la introducción de su compilación Martínez y Huang (2011: 8), un proyecto de este tipo no busca una definición estática y clara sobre práctica, sino que reconocen su pluralidad epistémica y lo que intentan es darle un significado epistemológico a tal concepto para explicar con mayor profundidad el proceso de la ciencia y la tecnología.

\section{La propuesta de la epistemología de la imaginación}

En cuanto a la epistemología de la imaginación (Rodríguez-Salazar 2011; 2015; 2016), que fue propuesta hace más de una década como epistemología de la acción (Hernández y Rodríguez, 2003), parte de una postura genética sin agotarse en ella, sino que va más allá. Desde esta postura se entiende la epistemología como una reflexión que emana de los propios científicos y ya no como una especulación filosófica, lo que indica su autonomía como una ciencia diferente a la filosofía de la ciencia.

Esta propuesta epistemológica, inició como un intento de responder cómo la imaginación es un recurso explicativo en el proceso de las configuraciones mentales que dan soluciones simbólico-materiales al problema del desarrollo de la ciencia. Derivado de ello, la epistemología es el estudio de las condiciones constitutivas [en el sentido piagetiano] del conocimiento desde una reflexión científica.

En ese sentido, la epistemología de la imaginación nace como una propuesta que hace énfasis en la acción y que inició estudiando los instrumentos en sus distintas conceptualizaciones.

Esto queda claro con la siguiente cita: 
[...] las modificaciones realizadas a los instrumentos se consideran como la materialización de la configuración imaginaria de una realidad posible. [...] los instrumentos son entonces una extensión, es decir, un despliegue hacia el exterior de las acciones evocadas de la experiencia simbólico-imaginativa a través de las acciones materiales de la experiencia práctica. Una vez construidos los instrumentos, éstos fueron generadores de nuevos repertorios de acciones. Como extensiones de las acciones, el planteamiento es que las acciones materiales llevadas a cabo con ellos se prolongan en la imaginación como nuevas acciones evocadas. Estas acciones, al ser desplegadas hacia el exterior, se materializan a partir de la creación de nuevos instrumentos o se materializan al modificar los instrumentos anteriores. (Rodríguez-Salazar, 2015: 105)

En efecto, el vínculo que une las acciones evocadas con las materiales es la experiencia simbólico-imaginativa que posibilita la creación de realidades posibles sobre cómo resolver los problemas de una realidad tangible. Así, el sujeto que observa el funcionamiento de algún artefacto (experiencia material) pasa a relacionarla con su bagaje de conocimientos y de experiencias, creando, de ese modo, imágenes o escenarios de realidades materialmente posibles (experiencia simbólico-imaginativa), para posteriormente diseñar algún producto que cumpla alguna función específica (experiencia formal).

De ese modo, Rodríguez-Salazar (2015) clasifica a los instrumentos como sigue:

a) Desarrollo tecnológico toda instrumentación matemática o material aplicada a la producción de bienes materiales. Así estos desarrollos tecnológicos son un "bien abstracto utilizado en la producción de bienes materiales." (p. 142), lo que implica que sólo cuando un instrumento tiene como fin el flujo económico es considerado como desarrollo tecnológico, es decir, hay una componente de mercado o empresarial.

b) Herramientas metodológicas, se refieren a aquellos instrumentos que soportan o apoyan el desarrollo de la investigación científica. Como en el ejemplo propuesto: "los instrumentos materiales, el versorium es el ancestro del electrómetro en tanto herramienta metodológica como instrumento de medición. En cuanto a los instrumentos matemáticos, la ecuación desarrollada por Jean-Baptiste Biot y Félix Savart representa una estructuración algebraica análoga” (p. 146). Con esa cita queda claro el concepto de 
herramienta metodológica, pues el versorium fue un instrumento utilizado por Gilbert para experimentar y comprobar los flujos magnéticos, mientras que la ecuación BiotSavart se utiliza para calcular la intensidad de corriente, lo que permitió, y aún permite, realizar operaciones con las cuales avanzar en investigaciones sobre electromagnetismo.

c) Instrumento científico es aquel que contribuye en el desarrollo científico que permite develar nuevos fenómenos antes ocultos. Es decir, a través de los instrumentos científicos se permite una estructuración epistemológica de las manifestaciones de nuevos fenómenos, que únicamente a través de estos instrumentos, pueden ser experimentados, sensibles al sujeto.

Ahora bien, si su planteamiento inicial fue sobre los instrumentos, la epistemología de la imaginación no se detuvo ahí y sigue su desarrollo teórico que se extiende a múltiples campos de conocimiento, como en psicología, matemáticas, física, medicina, entre otros.

Sin embargo, es lícito preguntarse: desde este enfoque epistemológico ¿cómo se entiende el concepto de imaginación?, bien pues esta parte de la idea kantiana de las formas puras del entendimiento, donde la imaginación, es un esquema libre de experiencia que, por un lado, es concebido como un pensamiento racional y, por el otro, juega un papel mediador entre intuiciones y conceptos (Rodríguez-Salazar, 2016: 82-83).

En esa misma línea kantiana, la imaginación es productiva y una facultad que sintetiza $a$ priori el conocimiento. De ese modo, la imaginación ya no es producto de la fantasía o de la especulación metafórica y filosófica de la realidad, sino, el ápice del conocimiento que configurará realidades posibles para devolverse a lo material en forma de solución.

Por otro lado, esta forma de entender la imaginación aún queda limitada, por lo que Rodríguez-Salazar (2015; 2016) retoma de Piaget las etapas del desarrollo psicogenético y los tres conjuntos de acciones que dan cuenta de la relación sujeto-objeto, las cuales son: 1) materiales, referentes al acto del sujeto sobre los objetos de la realidad; 2) evocadas, prolongaciones mentales de las acciones materiales que configuran la realidad; y, 3) operatorias, organización de la realidad exterior mediante representaciones formales.

La propuesta de la epistemología de la imaginación asume una noción ampliada de experiencia integra lo simbólico en esta relación, sostiene "que los tres conjuntos de acciones coexisten en todo sujeto y continúan funcionando coordinadamente durante toda 
la vida, conformando una estructura general de comportamiento cognitivo, o sea una estructura cognitivo-comportamental" (Rodríguez-Salazar, 2015: 164). Esta nueva forma de relación se muestra en la siguiente figura:

$$
\frac{M A O}{M A M} M A E \leftrightarrow \frac{A}{R} \leftrightarrow C I R P \frac{C I E F}{P I R}
$$

FIGURA 1. Propuesta de Rodríguez-Salazar de la relación Sujeto-Objeto bajo la noción ampliada de experiencia. Donde las siglas significan $\mathrm{MAO}=\mathrm{Mecanismo}$ de Acciones Operatorias; MAM= Mecanismos de Acciones Materiales; MAE $=$ Mecanismos de Acciones Evocadas; $\mathrm{A}=$ Acciones; $\mathrm{R}=$ Realidad; $\mathrm{CIRP}=$ Configuraciones Imaginarias de Realidades Posibles; CIEF= Configuración en Imágenes de Estructuras Formales; PIR= Propiedades Intrínsecas de la Realidad. . Fuente: Adaptación propia de la figura presentada en Rodríguez-Salazar (2015:165)

Así, estas acciones evocadas tienen un valor epistemológico que consiste en "crear las configuraciones imaginarias de realidades materialmente posibles" (p. 167). Por tanto, se interpreta en este trabajo que la imaginación es el puente entre lo mental y lo social.

Asimismo, de acuerdo con Rodríguez-Salazar \& Rosas-Colín (2011) existe una tríada cognitiva que, a través de la coordinación de sus esferas, hace posible la estructuración de la realidad. Esta se compone de Razonamiento Práctico (RP), Razonamiento simbólico-imaginativo (RSI) y Razonamiento Formal (RF). De ese modo, se puede argumentar que con la coordinación y comunicación de las esferas que componen la tríada cognitiva y con los mecanismos de acciones se pueden desarrollar en el niño esquemas y estructuras fortalecidas y ampliadas en la experiencia simbólica para pasar, en lo sucesivo, a la formalización de dichas imágenes.

De ese modo, el sujeto individual arriba al mundo social. Es decir, a través de estas configuraciones mentales el sujeto organiza una realidad socialmente aceptada, lo que implica que la subjetividad se objetiva a través de dicha organización. En ese sentido, se observa que la epistemología de la imaginación ofrece un modelo con el cual comprender la realidad social a través de las acciones con las que estructura la realidad el sujeto. 
En efecto, Rodríguez-Salazar (2016: 89) argumenta que esta estructuración del sujeto "Llevado al terreno social y en el marco de la epistemología genética, Piaget establece un paralelismo entre las estructuras de la inteligencia práctica y de las operaciones formales, con las estructuras de los grupos sociales."

De ese modo, Rodríguez-Salazar \& Rosas-Colín, (2011) sostienen, desde una postura neo-piagetiana, que el pensamiento, en tanto que forma y función, son de carácter social mientras que el contenido del pensamiento es individual. En este trabajo se sostiene que si bien el factor social no es determinante para la estructura y función del pensamiento adquiere un carácter co-evolutivo con la cognición que tiene como intermediario lo simbólico-imaginativo.

De ese modo, el lenguaje forma parte esencial en la estructuración del conocimiento. Puesto que el lenguaje simbólico individual adquiere una significación socialmente aceptada cuando se da esta dinámica co-evolutiva.

Por otro lado, un aspecto a tomar en consideración es lo referente a la observación. En ese tenor, el autor de la epistemología del imaginación, en su propuesta de epistemología de la acción, argumenta que lo que se observa siempre la coordinación de las acciones del sujeto sobre el objeto (Hernández \& Rodríguez, 2003: 83-124). Por lo tanto propone que la observación no es pura, pero también difiere que sólo sea una observación cargada de teoría. Lo que implica que, si bien el sujeto tiene esquemas mentales previos que le permiten organizar la realidad que conoce, tampoco observa la esencia del objeto, sino sus acciones dirigidas al objeto. Es decir, no existe observación pura, pero tampoco pura observación.

En cuanto al método que propone la epistemología de la imaginación, esta recupera el psicogenético y el factor social para configurar lo que denomina como método psicosociogenético (Rodríguez-Salazar \& Rosas-Colín, 2011: 46)

Por tanto, con base en todo lo planteado hasta ahora, se reconoce en este trabajo la pluralidad y, por tanto, en sintonía con el objetivo de esta trabajo, los enfoques epistemológicos que acabamos de describir se proponen como una propuesta a considerar como fundamentos filosófico-epistemológicos para las ciencias sociales. Por lo tanto, se considera que esta propuesta puede aportar a la epistemología en sus componentes 
interaccioncitas entre, no sólo lo cognitivo, sino, también, lo psicológico con lo social a través de lo simbólico-imaginativo.

De ese modo, la epistemología de la imaginación, por su carácter productivo, encuentra sus dominios materiales muy diversos en su aplicación, abarcando áreas de conocimiento desde la sustentabilidad hasta la economía, de la psicosociología hasta la matemática y la lógica, pasando por la biología y la pedagogía (Cf. Hernández \& Rodríguez-Salazar, 2003; Rodríguez-Salazar y Rosas-Colín 2011; Rodríguez-Salazar \& Díaz Barriga Arceo, 2018).

\section{Reflexiones finales}

La tesis del paralelismo ramificado de la cognición descrita por Martínez, es una versión, más o menos, coincidente con los planteamientos piagetianos y neo-piagetianos, puesto que son conceptualizaciones que toman la relación sujeto-objeto desde un paralelismo y un isomorfismo. Así la cognición no es pura estructuración mental pero tampoco se determina con la agencia o con lo social.

Otro punto de encuentro entre estos enfoques epistemológicos es el que trata sobre la observación. Para Martínez \& Huang (2011), coinciden con el argumento que la observación pura no existe; es decir, no es posible únicamente tener sensaciones del objeto sin intermediación de algo más. Este algo más es la configuración teórica del sujeto que observa. Así cuando dos científicos observan un mismo fenómeno pueden interpretarlo de distintas maneras puesto que lo observan desde enfoques teóricos distintos. Por otro lado, Hernández \& Rodríguez (2003) van más lejos, casi una década antes, retomando el planteamiento mismo de la inexistencia de la pura observación, y profundizan al argumentar que lo que existe es una observación de la coordinación de las acciones del sujeto sobre el objeto y los resultados de dicha acción. Este planteamiento coincide con ejemplo que exponen Shapin y Shaffer (2005) sobre la bomba de vacío: mientras que para Boyle observaba el vacío como un componente únicamente científico, Hobbes observaba en ese experimento del ingenio una justificación eclesiástica del poder. Por tanto, ambos podrían tener razón desde sus distintos enfoques.

Las diferencias sustanciales (si las podemos llamar así) de estos enfoques, se basan en el enfoque de las implicaciones que tiene cada versión. Mientras que para la epistemología 
de la imaginación, como enfoque post-piagetiano, tiene implicaciones directas tanto en la organización social y en los mecanismos de desarrollo y equilibrio del sujeto, como en las condiciones del conocimiento en general, para Martínez la cognición tiene implicaciones en el desarrollo tecnológico, especialmente en lo relativo a la inteligencia artificial, o bien, en la estructura normativa de la epistemología enfocada al conocimiento científico como un argumento de racionalidad, justificando de alguna manera al planteamiento kuhniano.

Otra de las diferencias que es importante hacer notar, es que el planteamiento de la epistemología naturalizada social de Martínez parte de una irreductibilidad social de la cognición, mientras que para la epistemología de la imaginación existen diferencias entre forma y función con el contenido cognitivo en lo individual y lo colectivo. Es decir, la primera parte de lo social hacia lo cognitivo, mientras que el planteamiento de la segunda parte de un proceso cognitivo individual que se socializa, desde el punto de vista psicogenético, el cual se puede extender hasta lo sociogenético, con el arribo del adolescente al mundo social. Lo mismo sucede en el terreno científico, con las propuestas individuales de cambio teórico, hasta su aceptación por la comunidad científica.

Por lo tanto, queda clara una cierta colaboración epistemológica para lo que atañe a este trabajo. En concreto, desde un marco plural, la epistemología naturalizada social, permite observar el valor explicativo del concepto de práctica para dar cuenta de cuestiones, tanto normativas, como contextuales o situadas, o lo que es lo mismo, una epistemología cercana a los procesos sociales. Por su parte, la epistemología de la imaginación de base psicogenética y sociogenética, por su carácter sincrónico y diacrónico, da cuenta de la estructura del pensamiento del sujeto y de su relación con lo social a través del pensamiento racional que media entre intuiciones y conceptos. También, este último enfoque tiene la posibilidad de fungir como amalgama entre la epistemología y otras disciplinas en una interrelación activa de conocimiento.

Así los criterios que nos ofrece Kuhn sobre la elección de la teoría podemos ubicar que ambos enfoques asignan un valor especial a la coherencia interna y externa de las teorías y a la simplicidad en cuanto su explicaciones en tanto que se acepta un grado de pluralidad. En cambio, la precisión es un valor más cercano a la epistemología de la imaginación en tanto que su carácter es más cercano a los procesos científicos, pues la 
propuesta de las prácticas se desenvuelve en una reflexión más especulativa. En tanto que fecundidad y amplitud ambos enfoques asignan un peso importante, sin embargo, la epistemología de la imaginación encuentra dominios materiales más amplios y fecundos (ver Tovar-Sánchez en este mismo libro) a no sólo limitarse al aspecto cognitivo, sino que explora áreas más extensas como el psicosocial, el económico, la matemática, en especial el de la psicología de la ciencia, que es motivo de otro trabajo.

\section{Referencias}

Hernández, G. \& Rodríguez, L. M. (2003). Observación y acción en el conocimiento científico. En Hernández \& Rodríguez-Salazar (coord.) Filosofía de la experiencia y ciencia experimental. (83-124) México: Fondo de Cultura Económico.

Kuhn, T. S. (1962/1985). La Estructura de las Revoluciones Científicas. México: Fondo de Cultura Económico.

Kuhn, T. S. (1977/1996) La Tensión Esencial. Estudios selectos sobre la tradición y el cambio en el ámbito de la ciencia. México: Fondo de Cultura Económica.

Martínez, S. (2005). Ciencia, valores y prácticas científicas. Actas del XII Congreso Nacional de Filosofía, AFRA/Universidad Nacional del Comahue, 1-9.

Martínez, S. (2009). Hacia una racionalidad ecológica. En Á. Eraña, G.

Martínez, S. (2011) La cognición coporizada en prácticas: implicaciones para la filosofía de la ciencia. En Martínez, S., Huang, X., \& Guillaumin, G. (compiladores). Historia, prácticas y estilos en la filosofía de la ciencia. Hacia una epistemología plural. (pp. 217 234) México, D.F.: Miguel Ángel Porrua.

Martínez, S. \& Huang, X. (2011) Introducción. Hacia una filosofía de la ciencia centrada en prácticas. En Martínez, S., Huang, X., \& Guillaumin, G. (compiladores). Historia, prácticas y estilos en la filosofía de la ciencia. Hacia una epistemología plural. (pp. 563) México, D.F.: Miguel Ángel Porrua.

Mayorga Madrigal, A. C. (2013) Una aproximación a los críticos de la epistemología genética. En Mayorga Madrigal, Ramírez González \& Ortiz Gutiérrez (coord.) Piaget en 
la actualidad. Reflexiones sobre epistemología genética. (21-40) México: Universidad de Guadalajara

Olivé, L. (2006). El bien, el mal y la razón. Facetas de la ciencia y de la tecnología. México, DF: Paidós Mexicana.

Piaget, J. (1967/1979b). Tratado de lógica y conocimiento científico, Tomo VII. Buenos Aires: PAIDÓS. Risjord, M. (2014). Philosophie of Social Science. New York: Taylor \& Francis.

Rodríguez-Salazar \& Rosas-Colín (2011) Bases teórico-metodológicas de una epistemología de la imaginación: ¿por qué Piaget? En Rodríguez-Salazar, QuinteroZazueta \& Hernández-Ulloa (coord.) Razonamiento Matemático Epistemología de la Imaginación (Re)pensando el papel de la epistemología en la Matemática Educativa. (3392) Barcelona, España: Gedisa.

Rodríguez-Salazar, L. M. (2015) Epistemología de la imaginación. El trabajo experimental de Ørsted. México: Corinter.

Rodríguez-Salazar, L. M. (2016) La imaginación en Kant y la epistemología de la imaginación. En Monroy-Nasr \& Rodríguez-Salazar (Editores) Imaginación y conocimiento de Descartes a Freud. (75-96) México: Corinter

Rodríguez-Salazar, L. M. \& Rosas-Colín, C. P. (2016) ¿Qué hacer en metodología de la ciencia? Entre la epistemología y la filosofía de la ciencia. En de Hoyos Bermea \& Riquelme Alcantar (coords.) Estudios sobre metodología de la ciencia: su impacto en la teoría y la práctica. (39-56) México: IPN

Rodríguez-Salazar, L. M. \& Díaz Barriga Arceo, F. (2018) Al este del paradigma: Miradas alternativas en la enseñanza de la epistemología. México: Gedisa

Shapin, S., \& Shaffer, S. (2005). El Leviathan y la bomba de vacío: Hobbes, Boyle y la vida experimental. Buenos Aires: Universidad de Quilmes Editorial. 